\title{
3D Cluster-Based Ray Tracing Technique for Massive MIMO Channel Modeling
}

\author{
M. M. Tamaddondar ${ }^{1}$, N. Noori ${ }^{2}$ \\ ${ }^{1,2}$ Department of Communication Technology, Iran Telecommunication Research Center, Tehran, Iran. \\ Corresponding author: Email: \{tamaddondar,nnoori\}@ itrc.ac.ir
}

\begin{abstract}
In this paper, a novel 3-dimensional (3D) approach is proposed for precise modeling of massive multiple input multiple output (M-MIMO) channels in millimeter wave $(\mathrm{mmW})$ frequencies. This model is based on both deterministic and statistic computations to extract characteristics of the propagation channel. In order to increase algorithm execution speed, the physical channel is divided into two regions. The first region refers to those parts of channel which can be mapped with simple planes such as walls, ramps and etc. The second region is usually complex which is defined by considering the channel with physical clusters. These physical clusters yield multipath components (MPCs) with similar angles of arrival (AoA) and time delay. The ray tracing algorithm is utilized to find ray paths from transmitter (Tx) to receiver (Rx). Some characteristics of MPCs in each cluster are defined according to some appropriate statistical distribution. The non-stationary property of M-MIMO along the antenna array axis is considered in the algorithm. Due to the correspondence between propagation environment and scatters, the accuracy of the model is highly increased. To evaluate the proposed channel model, simulation results are compared with some measurements reported in the literature.
\end{abstract}

INDEX TERMS Channel Modeling, Cluster, Massive MIMO, Stochastic Modeling.

\section{INTRODUCTION}

The fifth generation $(5 \mathrm{G})$ of wireless communication systems has attracted extensive amount of research efforts and attentions. The $5 \mathrm{G}$ technology can present extremely low latency, high energy efficiency, and very high data rate [1]-[5]. To achieve 5G design targets, information theory suggests three main key approaches $[2,6]$ : (i) ultradense networks which are known as small cell technology, (ii) large quantities of new bandwidth which means utilizing higher frequencies, and (iii) high spectrum efficiency by using numerous antennas at the base station (BS) refers to massive multiple input multiple output (M-MIMO). The antenna configuration in M-MIMO systems includes hundreds or even thousands of antenna elements to increase the channel capacity, improve spectral and energy efficiency and promote reliability of the system with respect to the conventional MIMO [7]-[16]. The M-MIMO systems may confront with non-stationary properties across the array axis. This phenomenon is usually neglected in the case of conventional MIMO channels [17]. Thus, different antenna elements may face distinctive propagation characteristics. Due to the large dimension of the antenna array, a receiver may be located in a shorter distance than Rayleigh distance criteria [14]. In such a case, the far-field (FF) condition is not fulfilled, and a spherical wavefront has to be considered [15, $16]$.

Millimeter wave technology is one of the most effective solutions to achieve huge bandwidth for 5G systems [17][20]. High data rates of several gigabits per second can be easily obtained in $\mathrm{mmW}$ communications [21]-[23].
Consequently, a mmW M-MIMO system has a great potential to improve wireless access and throughput [6, 24]. Furthermore, very short wavelength of $\mathrm{mmW}$ frequencies helps to significantly reduce the M-MIMO array size. Additionally, the high gain of large number of antenna provided by M-MIMO systems is helpful to overcome the severe pathloss of mmW signals [25]. Since performance of $\mathrm{mmW}$ M-MIMO systems is greatly related to the propagation environment, channel models of $\mathrm{mmW}$ MMIMO systems are very necessary in system design. Even though many models such as WINNER II [26], WINNER+ [27], COST 2100 [28], IMT-A [29] and 3D SCM [30] have been presented for conventional MIMO systems, but these channel models cannot sufficiently cover new emerging mmW M-MIMO technology for 5G systems. In recent years, many efforts have been accomplished to extract the channel behavior in $\mathrm{mmW}$ and M-MIMO systems.

TABLE I. ChARACteristics OF SOME M-MiMO CHANNEL MODELS.

\begin{tabular}{|c|c|c|c|c|c|}
\hline Ref. & $\begin{array}{c}\text { Non- } \\
\text { Stationary }\end{array}$ & $\begin{array}{c}\text { Spherical } \\
\text { Wave }\end{array}$ & $\begin{array}{l}\text { Antenna } \\
\text { Topology }\end{array}$ & $3 \mathrm{D}$ & MMW \\
\hline [31] & $\sqrt{ }$ & $\sqrt{ }$ & - & $\sqrt{ }$ & - \\
\hline [32] & $\sqrt{ }$ & $\sqrt{ }$ & - & $\sqrt{ }$ & $\sqrt{ }$ \\
\hline [33] & $\sqrt{ }$ & - & Planar & $\sqrt{ }$ & - \\
\hline [34] & $\sqrt{ }$ & $\sqrt{ }$ & - & $\sqrt{ }$ & - \\
\hline [35] & - & - & - & $\sqrt{ }$ & $\sqrt{ }$ \\
\hline [36] & - & $\sqrt{ }$ & Planar & $\sqrt{ }$ & - \\
\hline [37] & $\sqrt{ }$ & $\sqrt{ }$ & Planar & $\sqrt{ }$ & - \\
\hline [38] & - & - & Linear & - & $\sqrt{ }$ \\
\hline
\end{tabular}


The birth-death behavior of the clusters across array aperture was observed in the measurement-based channel model presented in [39], where a virtual $40 \times 40$ planar antenna array was used at Tx side. An indoor M-MIMO channel measurements at different frequencies 11, 16, 28, and $38 \mathrm{GHz}$ were conducted in [40] by a large virtual uniform rectangular array. Furthermore, many geometrybased stochastic models (GBSMs) have been presented to model massive MIMO channels with new features [18], [41]-[43]. The METIS channel model is a map-based model in which the layout of the propagation environment is predefined and channel coefficients are computed based on it [43]. In [44], a 3D vehicle M-MIMO channel model has been proposed where the spherical wavefront with non-stationary conditions has been considered to statistically derive channel parameters. Another channel model has been proposed in [45], where the far-field (FF) signals are modeled by plane waves and near-field (NF) signals are modeled with spherical waves. Some cluster-based channel models are summarized in Table I.

In this paper, a novel 3D hybrid channel model is proposed for wireless communication systems with the capability of simulating M-MIMO mmW channels. Here, the modeling procedure consists of two successive deterministic and stochastic modes. The combination of both modes forms the final channel model. In the deterministic mode, all surfaces, such as walls, floors, ceilings, ramps and etc. are defined with their equivalent planes. These planes are imported to the simulator in the form of rectangles along with their four corners position. Then, the Line of Sight (LoS) and reflection paths between Tx array antenna elements and Rx users of MMIMO system are extracted, and the image theory is utilized to find the first and second order reflection paths. If a path is encountered by a surface, the transmission phenomenon is also considered in the calculations. In the stochastic mode, those parts of the channel that cannot be modeled in the deterministic mode due to their geometric complexities are defined based on the cluster concept. Accordingly, the MPCs arrive at $\mathrm{Rx}$ user with similar features of delay, and AoA make clusters. By means of appropriate statistical random distributions, the MPCs of the clusters are extracted and their characteristics such as phase shifts, intra-cluster delay, and their position within each cluster are modeled. The channel impulse response (CIR) is obtained from combination of two deterministic and stochastic modes. Other main characteristics of the channel such as delay spread, AoA and angle of departure (AoD) are then extracted. This proposed model not only has high accuracy, but also takes little computational time to model whole M-MIMO channel. To evaluate this model, simulation results are compared with some measurements reported in [46].

The major contributions of this paper are summarized as follows: The proposed model can be applied to a wide range of propagation channels. Since the modeling procedure is performed in two successive deterministic and stochastic modes, it can adopt to the real propagation environments, despite its simplicity of implementation. Also, the nonstationary property of the channel can be modeled along the M-MIMO antenna array in this model.

The following sections of this paper are organized as follows. Section 2 describes fundamental of the proposed channel model for non-stationary M-MIMO channels. Theoretical and mathematical details of the proposed channel model are explained in Sec.3. The scenario description is presented in Sec.4. Some simulation results and discussions are given in Sec.5. Finally, conclusions are presented in Sec.6.

\section{DESCRIPTION OF THE CHANNEL MODEL}

At first step, geometrical boundaries of the propagation environment as well as walls, ceiling and floor are defined and their electromagnetic properties are specified. Furthermore, $\mathrm{Tx}$ and $\mathrm{Rx}$ antenna positions and their characteristics such as radiation pattern, array elements configuration and polarization are defined. Other parameters such as frequency and transmit power are also specified. The desired channel parameters are defined at this step to be extracted at the end of the simulation procedure. At the next step, channel simulation is performed according to the predefined propagation environment and simulation strategy. To overcome the complexity of the channel, this step is divided into two deterministic and stochastic modes as mentioned before.

\section{A. Deterministic modeling}

Deterministic modeling considers those parts of the channel that can be defined as planes. If these parts are located in the FF region, the FF approximation is considered to compute the amplitude and phase shift of each path. Otherwise, the $\mathrm{NF}$ conditions are considered in calculations.

The $3 \mathrm{D}$ ray tracing algorithm is used in this stage to find propagation paths between the Tx and Rx. Two important propagation phenomena (reflection and transmission) are considered in deterministic modeling. According to Fig. 1, by utilizing image theory, the reflection points on each wall are obtained. The incident angle of each path and electromagnetic characteristics of the obstacles defined at the first step, are used to calculate Fresnel reflection and transmission coefficients according to the polarization.

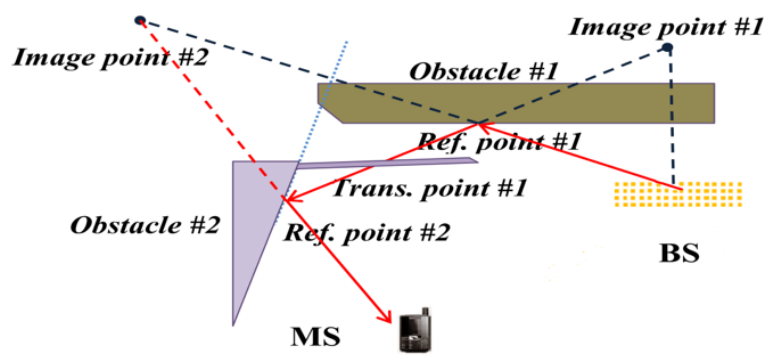

Figure 1. Schematic of ray tracing algorithm. 
These reflected and transmitted paths are considered to calculate the intensity of the received signal. The whole procedures are done between each Tx element and all users. This algorithm is continued until all defined deterministic parts are checked to find existence (or non-existence) of a path between each Tx antenna and every user. Then, the algorithm runs out to switch to the stochastic modeling (if necessary).

\section{B. STOCHASTIC MODELING}

Generally, deterministic modeling of the whole channel is very complicated for M-MIMO systems. To overcome this complexity, a cluster-based stochastic modeling is proposed to complete the previous step and improve model accuracy. The cluster-based model is shown in Fig. 2. In this model, those parts of the channel that modeled stochastically are mapped by clusters.

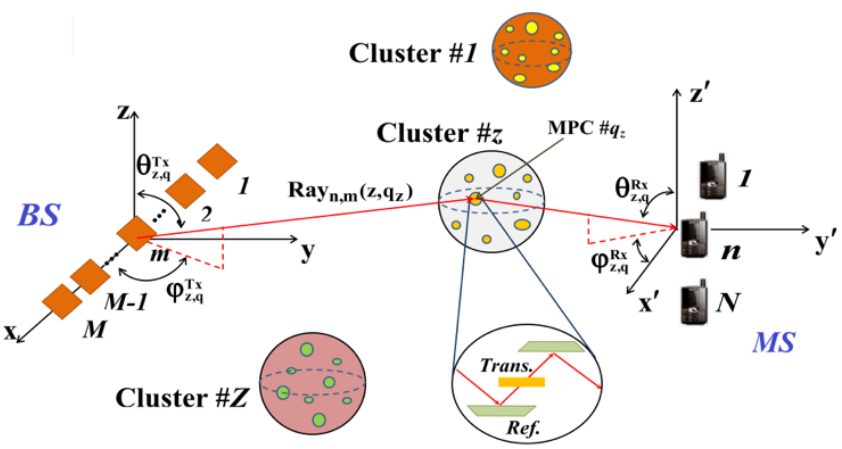

Figure 2. An overview of cluster-based M-MIMO channel model.

The stochastic modeling is based on the clustering behavior of the propagation channel since the MPCs reach the Rx in the form of bundles of rays with similar properties such as delays and AoAs. The stochastic parts of the channel are defined as shown in Fig. 3a and called interacting objects (IOs). These interacting objects are fences, crowds of vehicles, vegetation and so on that cannot be modeled in the deterministic mode due to the complexity of their geometry and the lack of conditions for utilizing ray tracing algorithm. The physical boundaries of these parts are then determined, as shown in Fig. 3 b. In order to simplify the calculations, equivalent spheres are embedded in the specified area instead of IOs, which have the role of meshing in the calculations of the stochastic mode. The configuration of the equivalent spheres can have a uniform or non-uniform structure similar to that shown in Fig. 3 c. Then, the space around the Rx is divided into cluster cells as shown in Fig. 3 d. If the cluster cells have physical intersection with the equivalent spheres, then we will have an active cluster cell. Several active cluster cells are shown in Fig. 3 d. The MPCs are assigned to the active cluster cells as shown in Fig. 3 e.

The phenomena of reflection and transmission occur in the cluster and other components are neglected in the calculations due to their low amplitude level.
All MPCs experience extra phase shifts and delays in the cluster that need to be considered. The phase shifts of each MPC can be any amount in the interval of $[0,2 \pi)$. A uniform distribution is proposed to model phase shift behavior of each MPC. The number of MPCs in each cluster is modeled by Poisson distribution with mean value of 10 . An exponential distribution is used to model the intra-cluster delay time.

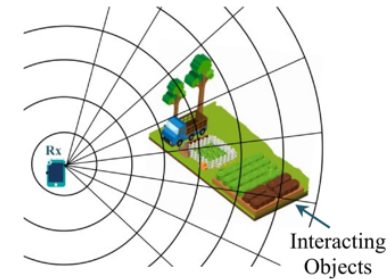

(a)

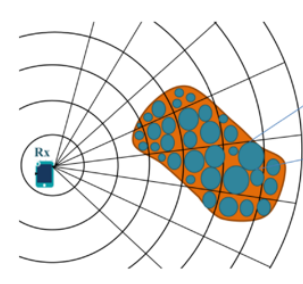

(c)
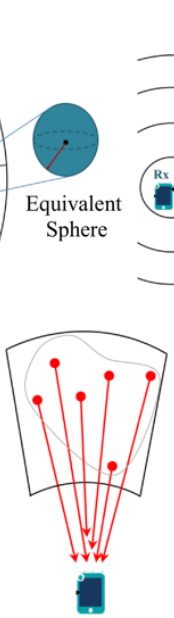

(e)

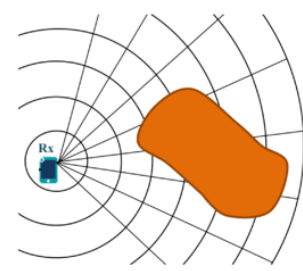

(b)

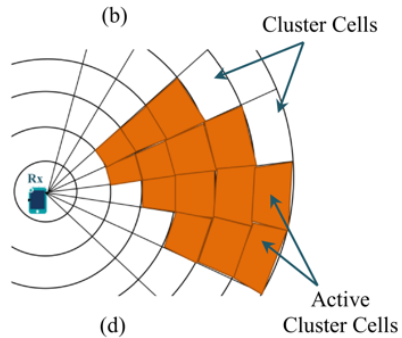

(d)

Figure 3 . The process of modeling objects in stochastic mode, a) Rea interacting objects (IOs), b) Cross section of the overall area, c) Modeling the IOs with equivalent spheres, d) Active clusters, e) MPCs produced in an active cluster cell.

Table II. Some proposed random distributions for channel modeling.

\begin{tabular}{|c|c|}
\hline Variable & Distribution \\
\hline $\begin{array}{c}\text { Number of intra-cluster } \\
\text { MPCs }\end{array}$ & Poisson [47] \\
\hline Phase shift of MPCs & Uniform [48] \\
\hline Time delay of MPC & Exponential [48] \\
\hline DOD \& DOA & $\begin{array}{l}\text { Wrapped Gaussian or } \\
\text { Von Mises [18], [49] }\end{array}$ \\
\hline
\end{tabular}

The mean value of the exponential distribution is chosen according to the physical size of the clusters in which the ray strength reduced significantly and here is considered to be about $2.5 \mathrm{~ns}$. These distributions are summarized in Table II.

\section{COMBINATION OF TWO STAGES}

As described in the previous subsections, the proposed channel model is executed in two stages. When both deterministic and statistic simulations are completed, the results should be post-processed. For this purpose, all MPCs 
received by the user are sorted according to their delays. The multipath delay axis $\tau$ is quantized into equal time delay segments called delay bins. Each bin has a time delay width equal to $\tau_{k+1}-\tau_{k}$ for $k=0$ to $K-1$, where $K$ represents total number of equally-spaced bins, and is determined by maximum delay of MPCs.

Any MPC received within the ${ }^{k}$ th bin is represented by a single resolvable delay ${ }^{\tau_{k}}$. All MPCs within delay bin of ${ }^{\tau_{k}}$ are summed up together to obtain signal strength at that bin. Then, desired characteristics of the channel are extracted based on processing of MPCs data. A general flowchart of the proposed channel model algorithm is illustrated in Fig. 4.

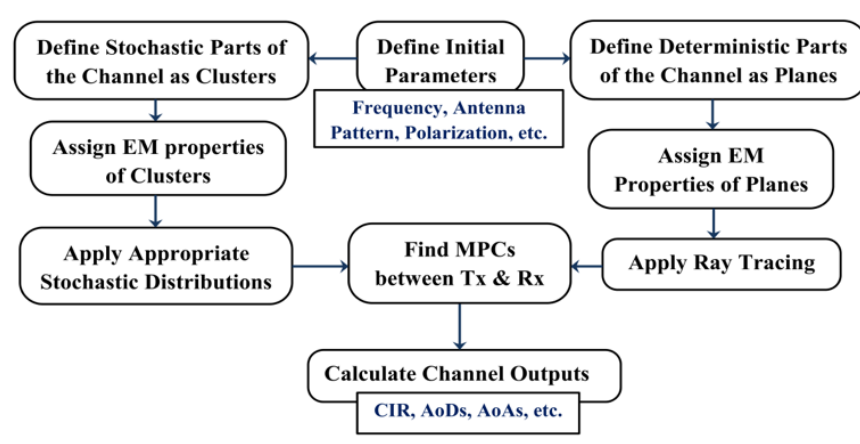

Figure 4. The flowchart of proposed channel model.

\section{THEORETICAL DETAILS OF THE PROPOSED CHANNEL MODELING ALGORITHM}

The MIMO channel is described by a matrix $H \in C^{M \times N}$, where the $\mathrm{BS}$ is equipped with $M$ antenna elements and serves $N$ single-antenna users. Each entry of the matrix $\mathbf{H}$ includes superposition of both deterministic and stochastic impulse responses. If the channel can be considered noiseless, the input-output relationship in the time domain can be expressed by:

$$
\mathbf{Y}(t)=\mathbf{H}(t) * \mathbf{X}(t)
$$

where $\mathbf{X}(t)$ is an $M \times 1$ vector of transmitted signals, $\mathbf{Y}(t)$ is an $N \times 1$ vector of received signal and operator $(*)$ denotes convolution. The channel matrix $\mathbf{H}$ is given by:

$$
\mathbf{H}(t ; \tau)=\sum_{k=1}^{K} H_{k} \cdot \delta\left(\tau-\tau_{\mathrm{k}}(t)\right)
$$

where $H_{k}$ is CIR of the $k$ th time bin denotes with $\tau_{k}(t)$.

\section{A. Mathematic theory of the deterministic algorithm}

In the deterministic algorithm, the normalized unit vectors of the surfaces defined in Sec.2.1 are used to identify each surface and compute reflection and refraction angles. Then, by applying image theory, the reflection point on each surface (if exists) is obtained.

After finding all paths between the $m$ th Tx element and the $n$th user, the entries of channel matrix $h_{m, n}^{d}$ are calculated as follows, where the superscript $d$ denotes to the deterministic part of the CIR:

$$
\begin{aligned}
h_{m, n}^{d}(t ; \tau) & =h_{m, n}^{L o S}(\mathrm{t} ; \tau)+\sum_{l=1}^{L} a_{l}^{d}\left(t ; \tau_{l}\right) \exp \left(-j \beta r_{l}\right) \\
& \times g_{m}^{T x}\left(\theta_{l}^{T x}, \phi_{l}^{T x}\right) \cdot g_{n}^{R x}\left(\theta_{l}^{R x}, \phi_{l}^{R x}\right) \cdot \delta\left(\tau-\tau_{l}(t)\right)
\end{aligned}
$$

where, the LoS component between the $m$ th Tx element and the $n$th $\mathrm{Rx}$ element is denoted with $h_{m, n}^{\text {LoS }}(\mathrm{t} ; \tau)$. The subscript $l \in\{1, \ldots, \mathrm{L}\} \quad$ denotes the $l$ th MPC in deterministic mode with amplitude of $a_{l}^{d}$. The parameter $\beta$ indicates propagation constant of the channel. The AoA in elevation and azimuth planes for the $l$ th ray are $\theta_{l}^{R x}$ and $\varphi_{l}^{R x}$, respectively, while the $n$th user's antenna gain is $g_{n}^{R x}$. The AoDs in elevation and azimuth planes are $\theta_{l}^{T x}$ and $\varphi_{l}^{T x}$, respectively, while the $m$ th $\mathrm{Tx}$ antenna gain is $g_{m}^{T x}$. The amplitude attenuation $a_{l}^{d}(t ; \tau)$ at time $t$ and delay $\tau$ is defined according to the path length and number of transmissions and reflections experienced by the $l$ th MPC. It can be expressed as follows:

$$
a_{l}^{d}\left(t ; \tau_{l}\right)=\left(\lambda / 4 \pi r_{l}\right) \prod_{u=l}^{U} \prod_{v=l}^{V} R_{v, l} T_{v, l}
$$

where $R_{v, l}$ and $T_{v, l}$ are reflection and transmission coefficients regarding to the $v$ th reflection and the $u$ th transmission experienced by the $l$ th MPC. The parameter $r_{l}$ is the distance travelled by the $l$ th MPC.

\section{B. Mathematic model of stochastic algorithm}

Interacting objects modeled in the stochastic mode are replaced by equivalent spheres. The configuration of the equivalent spheres is similar to the structure of the IOs. Then, active clusters are identified through the intersection detection algorithm of equivalent spheres and clusters. Although equivalent spheres can have variable centers and radii, the following limitations must also be considered:

- Equivalent spheres are just defined in the area of IOs,

- Depending on the distance from the $\mathrm{Rx}$ and the dimensions of the cluster cells, the radius of the equivalent spheres is variable, as shown in Fig. 3,

- Each equivalent sphere should not occupy more than one cluster cell,

- The equivalent spheres diameters should not be larger than IOs dimensions. 
According to the cluster parameters shown in Fig. 5, the following condition is considered to determine the radius of each equivalent sphere $R_{s p h}$ :

........... $\frac{\lambda}{2}<R_{s p h} \leq \min \left\{R_{s p h, 1}, R_{s p h, 2}, R_{s p h, 3}\right\} \ldots \ldots$

......where $\quad \cdots\left\{\begin{array}{l}R_{s p h, 1}=r_{c} \\ R_{s p h, 2}=(s-1) r_{c} \varphi_{c} \\ R_{s p h, 3}=(s-1) r_{c} \theta_{c}\end{array}\right.$

The range and azimuth and elevation angles of the $S$ th cluster cell are denoted by $r_{c}, \varphi_{c}$ and $\theta_{c}$ as shown in Fig.

5. The dimensions of the IOs are assumed larger than wavelength in the calculations. Thus, the minimum radius of the equivalent spheres is greater than half of the wavelength.

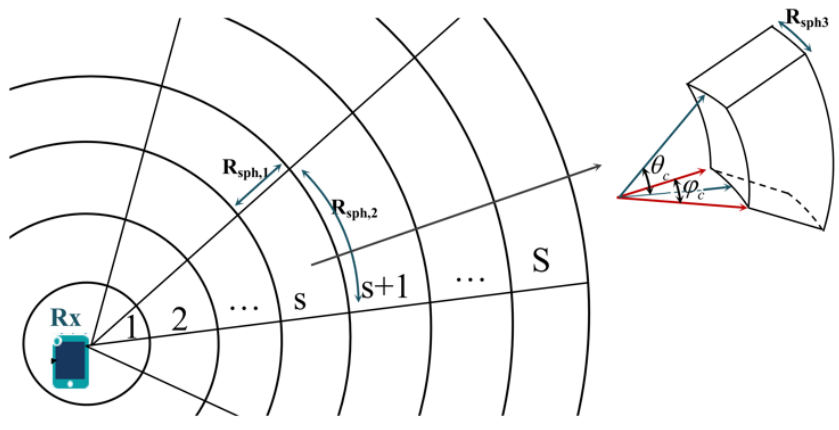

Figure 5. Cluster parameters.

Some appropriate statistical distributions are applied to extract characteristics of MPCs resulted from clusters. The entry of stochastic CIR matrix between the $m$ th Tx antenna and the $n$th user, $h_{m, n}^{c}$, is expressed as follows, while the superscript $c$ denotes the cluster-based calculations:

$$
\begin{gathered}
h_{m, n}^{c}(t ; \tau)=\sum_{z=1 q=1}^{Z} \sum_{z=q}^{Q_{z}} a_{z, q}^{c}(t ; \tau) \exp \left(-j\left(\beta r_{z, q}+\phi_{z, q}(t)\right)\right. \\
a_{z, q}^{c}(t ; \tau)=\left(\lambda / 4 \pi r_{z, q}^{c}\right) R_{z, q} T_{z, q}
\end{gathered}
$$

where parameter $z \in\{1, \ldots, Z\}$ and $q \in\left\{1, \ldots, Q_{z}\right\}$ are total number of active clusters and number of MPCs in the $z$ th active cluster, respectively. The amplitude of the $q$ th ray in the $z$ th cluster are denoted by $a_{z, q}(t ; \tau)$. Furthermore, $r_{z, q}$ and $\phi_{z, q}$ are the path length and excess phase shift of the $q$ th ray in the $z$ th cluster, respectively. The AoAs in elevation and azimuth planes are $\theta_{z, q}^{R x}$ and $\varphi_{z, q}^{R x}$, respectively for the $n$th user's antenna with gain $g_{n}^{R x}$. The
AoDs in the elevation and azimuth planes are $\theta_{z, q}^{T x}$ and $\varphi_{z, q}^{T x}$, respectively for the $m$ th $T x$ antenna with gain $g_{m}^{T x}$. Propagation delay of the $q$ th ray in the $z$ th cluster is indicated by $\tau_{z, q}^{c}$. Furthermore, $R_{z, q}$ and $T_{z, q}$ are reflection and transmission coefficients corresponding to the $q$ th ray in the $z$ th cluster, respectively.

The number of MPCs in $z$ th cluster, $Q_{z}$, is modeled with Poisson distribution as:

$Q_{z} \sim \operatorname{Pois}\left(\lambda_{p}\right)$ with $\left\{\begin{array}{l}\lambda_{p}=1, \text { for sparse environment } \\ \lambda_{p}=5, \text { for moderate environment } \\ \lambda_{p}=10, \text { for dense environment }\end{array}\right.$

The parameter $\lambda_{P}$ is a real number and equal to expected value of the number of MPCs in each cluster.

The delays of each MPC are calculated based on the length of the path that each MPC travels, while the angles of arrival to the receiver depend on the position of the each MPC in the cluster. In this way, in each cluster, a number of MPCs may reach the receiver. Therefore, according to the origin of the rays generated within each cluster, the vector between the points in the corresponding cluster to the receiver is obtained. Accordingly, the arrival angles are calculated in both azimuth and elevation planes. It should be noted that the scatters are positioned based on a uniform statistical distribution in each cluster. The phase of each path is also obtained according to the path length of each MPC, in addition to the amount of phase that is added after interaction within each cluster (which is modeled using a uniform distribution). However, in relation to the amplitude of each MPC, first, the path loss is obtained according to the Friis transmission equation and then, the attenuation coefficient in each cluster is added due to the propagation phenomenon that occurs in each interaction. Since there are closed-formed mathematic relations for reflection and transmission coefficient, these coefficients are taken into account in calculating the amplitude of these MPCs. Although these two phenomena may occur in many cases, the phenomena of diffraction and scattering are also very likely. However, diffraction and scattering phenomena do not have simple and closed-form mathematical equations, and on the other hand, in most cases, the power levels of such components are very small to be neglected in the calculations.

\section{Integration of deterministic and stochastic calculations}

All of the MPCs are classified into quantized delay time bins. The MPCs which have power more than threshold level are taken into account. Then, all MPCs of each delay time bin are summed up together. The power 
corresponding to each bin is calculated to determine the power delay profile (PDP). Accordingly, CIR of $k$ th interval $h_{m, n}^{k}$ can be presented as:

$$
h_{m, n}^{k}\left(t ; \tau_{l}\right)=\sum_{l=1}^{L_{k}^{d}} h_{m, n}^{d}\left(t ; \tau_{l}\right)+\sum_{l=1}^{L_{k}^{c}} h_{m, n}^{c}\left(t ; \tau_{l}\right)
$$

where $L_{k}^{d}$ and $L_{k}^{c}$ are number of deterministic and stochastic MPCs with delay $\tau$ in the $k$ th interval $\left.\left[\left(\tau_{k-1}+\tau_{k}\right) / 2,\left(\tau_{k+1}+\tau_{k}\right) / 2\right)\right]$, respectively. By quantizing CIR, received power from the $m$ th Tx antenna to the $n$th user can be expressed by:

$$
P R_{m, n}\left(t ; \tau_{l}\right)=10 \log _{10} P_{t}\left|h_{m, n}^{k}\left(t ; \tau_{l}\right)\right|^{2} \quad(\mathrm{dBW})
$$

where $P_{t}$ is transmit power. The mismatch and cable losses are neglected in (8).

Since AoAs of the MPCs in the respective time bin vary relative to each other, the AoA is defined individually for each MPC in the integration unit.

The root-mean-squared (RMS) delay spread can be calculated by using PDP. The RMS delay shows dispersion of delay and can be expressed as follow [46]:

$$
\tau_{r m s, m, n}=\sqrt{\frac{\sum_{P} P R_{m, n}\left(\tau_{p}\right) \tau_{p}^{2}}{\sum_{P} P R_{m, n}\left(\tau_{p}\right)}-\left(\frac{\sum_{P} P R_{m, n}\left(\tau_{p}\right) \tau_{p}}{\sum_{P} P R_{m, n}\left(\tau_{p}\right)}\right)^{2}}
$$

where $\tau_{p}$ and $\mathrm{PR}_{m, n}\left(\tau_{p}\right)$ are delay and received power of the $p$ th path between the $m$ th Tx antenna and the $n$th user, respectively.

Depending on the position of each antenna (assuming the phase center of each antenna which is related to the center position of that antenna), the ray tracing algorithm is applied separately to all of the antenna elements.

\section{SIMULATION ENVIRONMENT}

The wave propagation simulator is developed in MATLAB software. The simulator has a main engine that consists of several sub-functions. These sub-functions calculate different parts of the channel. Figure 6 shows the general structure of the simulator program. This program includes the section of initial definitions of IOs in both deterministic and stochastic modes such as, operating frequency, transmission power, polarization of the antennas, and the section of the calculation of the arrival and departure angles of each MPC, attenuation coefficients of each MPC and its phase, and finally a section which displays the results. The elapse time of the program strongly depends on the details of the propagation environment. However, since calculating the MPCs is performed in a computational procedure and is not based on the search algorithms, modeling in this method is much faster.

All simulations are conducted for the Center Hall in JiuJiao Teaching Building, Beijing Jiaotong University, China presented in [46].

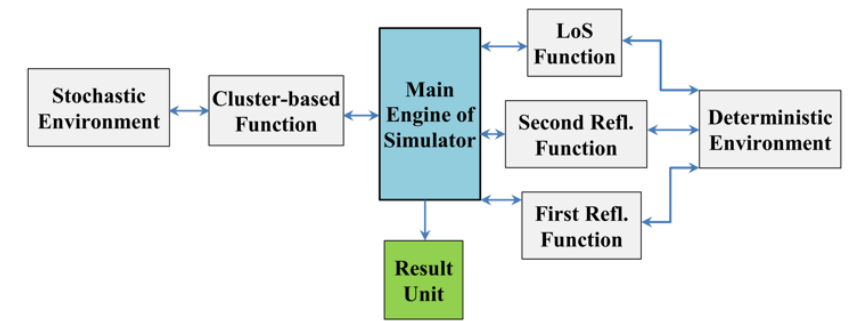

Figure 6. The structure of the simulator and sub-functions.

An overview of the simulated indoor environment is shown in Fig. 7. The length, width and height of the Hall are $20.1 \mathrm{~m}, 20.2 \mathrm{~m}$ and $4.5 \mathrm{~m}$, respectively. The platform is 0.8 $\mathrm{m}$ above the ground of the Hall, where Tx array antenna elements are placed $1.7 \mathrm{~m}$ above the center of the platform. $\mathrm{Rx}$ antenna is located in the mid of the hall at a distance of 4 $\mathrm{m}$ far from the center of the Tx antenna array. The height of $\mathrm{Rx}$ antenna is $2.0 \mathrm{~m}$ above the ground of the hall.

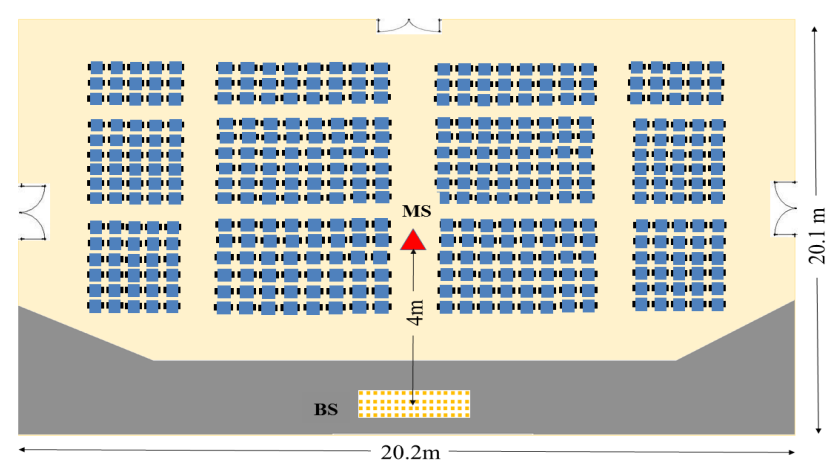

Figure 7. An overview of simulation environment.

The obstacles in the Hall are assumed to be fixed without any movement. The antenna array at Tx side includes 64 elements with uniform linear configuration parallel to the ground. The carrier frequency is set to 26 $\mathrm{GHz}$ during our simulations. The distance between successive antenna elements is equal to half of the wavelength. Transmit power is assumed to be $1 \mathrm{~W}(0$ $\mathrm{dBW}$ ) in the simulations. All Tx and Rx antennas have isotropic radiation patterns. The simulations begin with initial parameters given in Table III. In this table, the separation distance between array elements in the BS is denoted by $d_{e}$. Since the walls, ceiling, ground floor and the platform of the hall are all defined in the deterministic mode, the image theory is applied to them in order to find MPCs between Tx and Rx.

Then, characteristics of each MPC such as pathloss, phase shift, delay and AoAs are computed according to the mathematical formulations described in the previous section. Since the seats of the hall are too complex to model in 
deterministic mode, they are modeled in the stochastic mode. Then, appropriate statistical distributions as mentioned in Table II are used to model clusters and their MPCs. The threshold level is considered to be $-140 \mathrm{~dB}$ in the simulations.

Table III. Some initial description of simulation.

\begin{tabular}{|l|}
\hline Carrier Frequency : $26 \mathrm{GHz}$ \\
\hline BS Antenna : \\
64-Element, Linear Array, Isotropic Pattern \\
\hline BS Center Position: $[1.1,10.1,2.5]$ \\
\hline MS Antenna : \\
Single element, Isotropic Pattern \\
\hline MS Center Position: $[5,10,2]$ \\
\hline Transmit Power: $1 \mathrm{~W}$ \\
\hline
\end{tabular}

\section{RESULTS AND ANALYSIS}

First of all, according to Fig. 8, the walls, floor and ceiling are defined in the deterministic mode. The Tx has 64 antenna elements to cover a type of M-MIMO system. The LoS and reflection MPCs are found in this stage. The first and second order reflections are considered by applying image theory to all paths between each $\mathrm{Tx}$ array antenna element and $\mathrm{Rx}$ user. If any MPC is cut off by a surface, the transmission coefficient is also taken into account. Then, the remaining parts of the environment, such as hall seats, are defined in the stochastic mode as shown in Fig. 9.

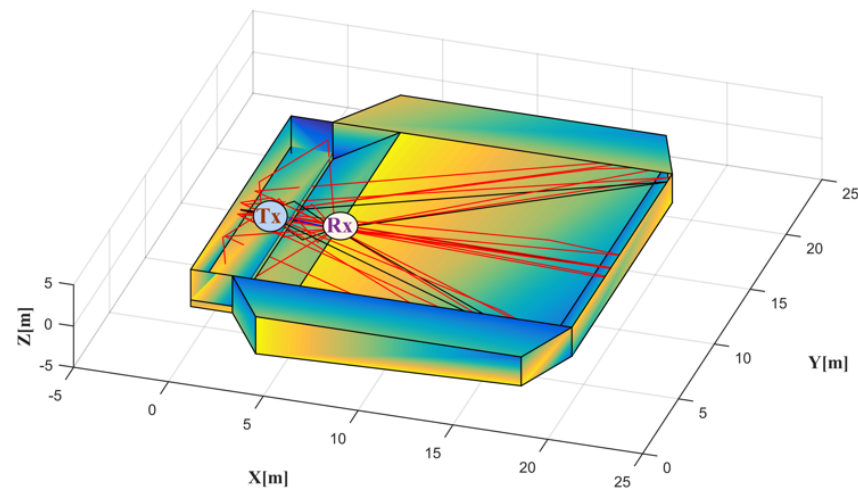

Figure 8. Deterministic mode of the channel model.

The aforementioned appropriate statistical distributions are deployed to model MPCs in the stochastic mode and extract their number, phase shift, and delay. Furthermore, other characteristic of the channel such as PDP, RMS delay spread, AoAs and AoDs are extracted by the combination of both deterministic and stochastic modes.

The PDP diagram between the first antenna element of the Tx array and the $\mathrm{Rx}$ is illustrated in Fig. 10. The measurement data extracted from the reported results in [46] is used in this figure to compare with our simulation. As illustrated by authors in [46], their measurement system includes a virtual antenna array at the Tx, while a single antenna is used at the Rx. Some more details of the measurement system are described in [46]. It can be seen that the peaks and nulls of the simulation model follow substantially the measurement results.

The RMS delay of the proposed channel model is compared in Fig. 11 with those of the measurement results reported in [46]. As it can be seen, the RMS delays fluctuate across the array antenna of M-MIMO system because the MPCs power changes along the axis of the array.

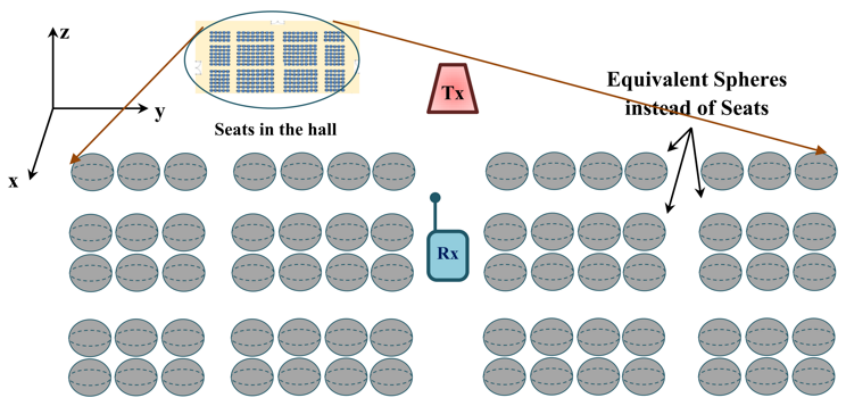

Figure 9. Equivalent spheres instead of stochastic parts (seats) of the channel.

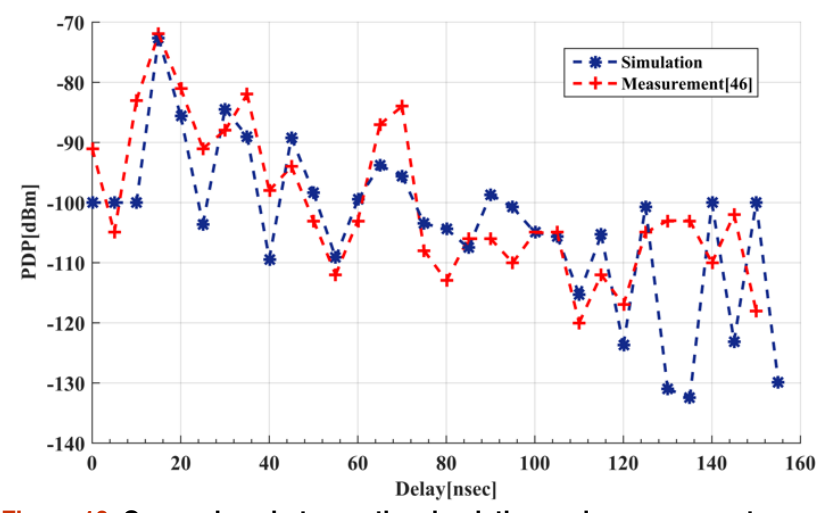

Figure 10. Comparison between the simulation and measurements.

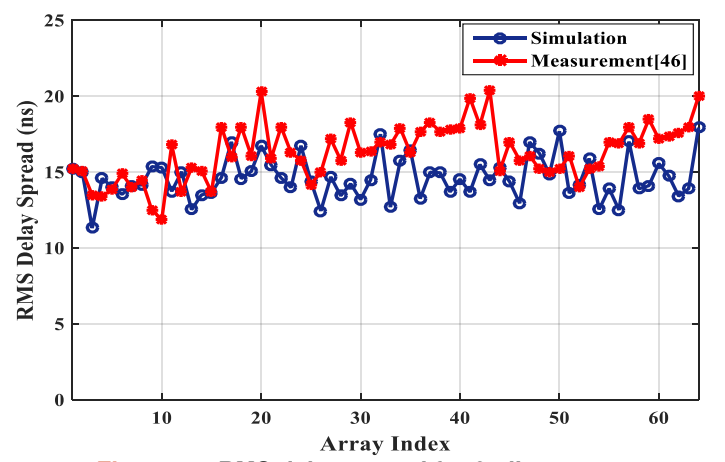

Figure 11. RMS delay spread for 64-linear array.

Figure 12 shows simulated PDP of the M-MIMO system along the 64-element array. It is observed that behavior of the LoS components for entire all array elements are the same, while the other MPCs arrived with different time delay. 


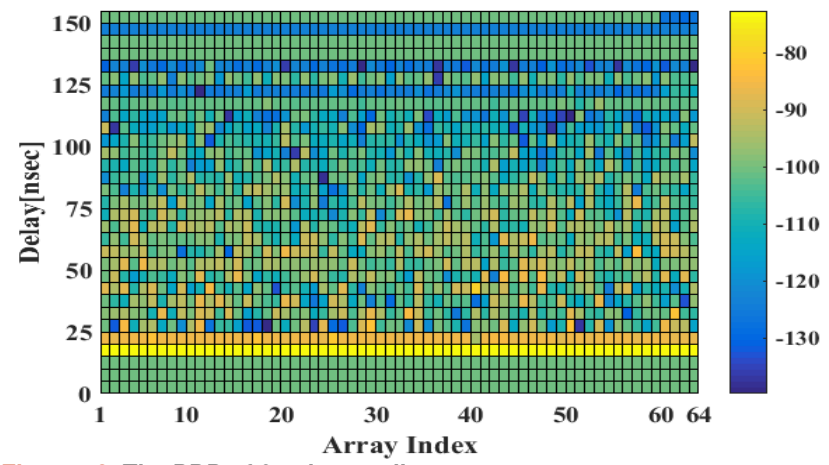

Figure 12. The PDP of 64-element linear array.

Figure 12 also illustrates the non-stationary property of NLoS components in the M-MIMO system.

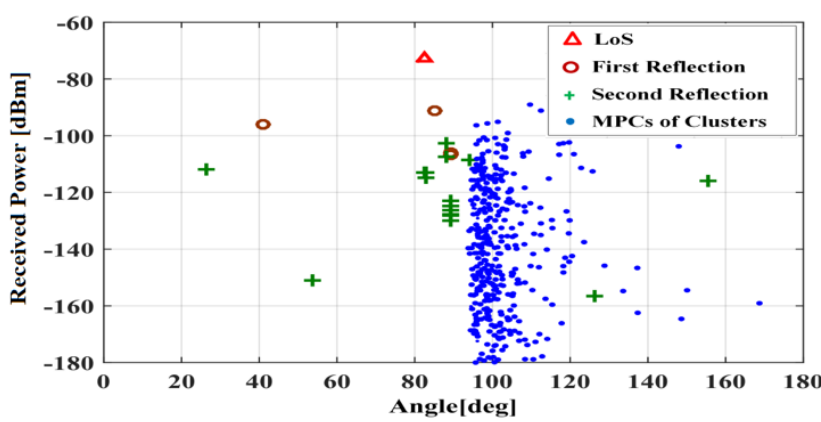

(a)

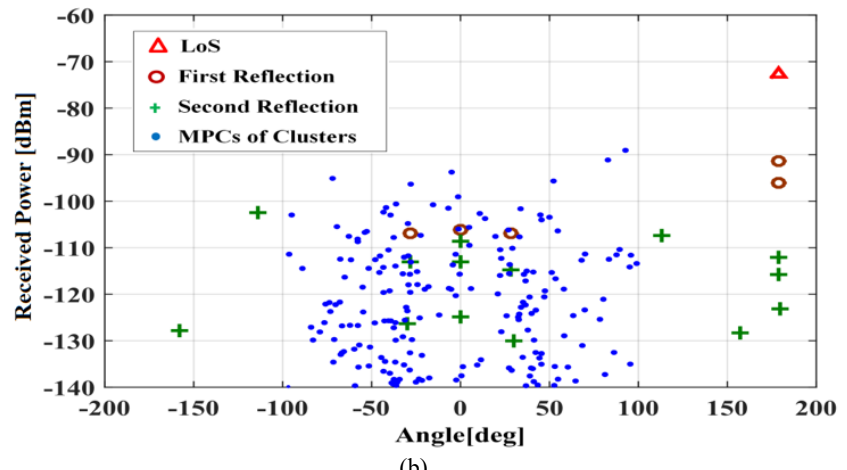

Figure 13. AoAs of MPCs, a) In elevation plane, b) In azimuth plane.

The AoAs of MPCs in the elevation and azimuth planes are shown in Fig. 13 a and Fig. 13 b, respectively for the first array element. Different kinds of MPCs are shown with various signs. It can be observed in Fig. 13 a, that accumulation of MPCs in elevation plane is around $100^{\circ}$ as it is expected. This is because of the Rx antenna is located at a higher level in the middle of the clusters. Furthermore, the MPCs within each cluster have a bit deviation in AoAs in the elevation plane. On the other hand, since the Rx antenna is located amid of the clusters, the AoAs in azimuth plane spread in a wide range of angles according to Fig. $13 \mathrm{~b}$.

The AoAs on the array axis in the elevation and azimuth planes are also illustrated in Fig. 14 a and Fig. 14 b, respectively.

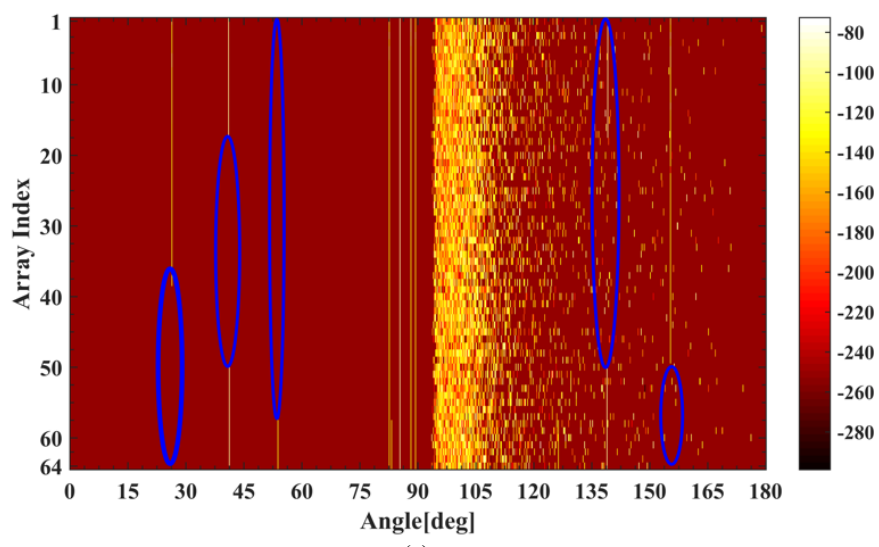

(a)

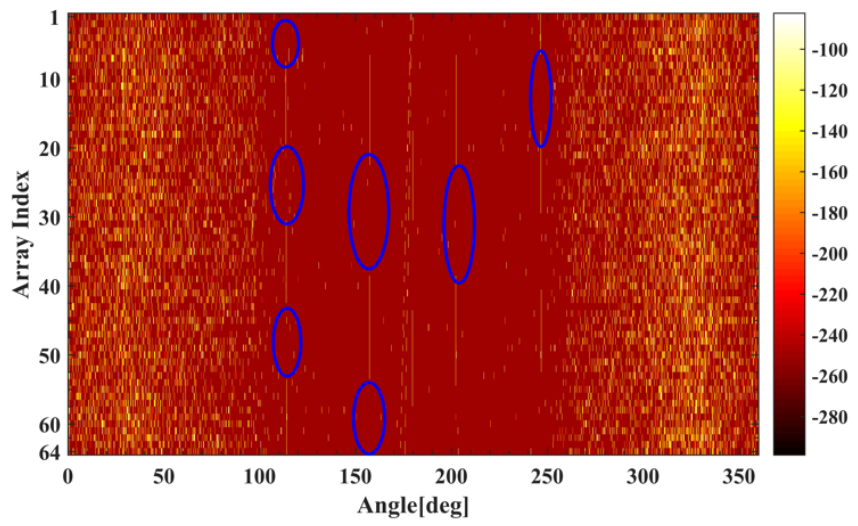

(b)

Figure 14. AoAs of 64-element array antenna, a) In elevation plane, b) In azimuth plane.

Again the fluctuation of the power strength and the (none) existence AoAs of the MPCs on the array axis in Fig. 14 a and Fig. $14 \mathrm{~b}$ show the non-stationary property of the MMIMO system. Some of these changes on the array axis are shown with elliptical shapes. It indicates that the M-MIMO antenna cannot be considered as single antenna element like conventional MIMO system.

\section{CONCLUSION}

A novel channel model for M-MIMO over mmW has been proposed in this paper for $5 \mathrm{G}$ networks. The proposed model is divided into two deterministic and stochastic regions. In the deterministic mode, the channel is defined for the simulator with details and by applying ray tracing algorithm, all propagation paths between $\mathrm{Tx}$ and $\mathrm{Rx}$ are determined. Then intensity and other characteristics of the received signals are obtained. But, since the M-MIMO channel is generally a very complex environment, the whole channel cannot be simulated in the deterministic mode. Thus, the second mode of the channel modeling algorithm is applied to characterize different objects located in the propagation environment. In this stage, those parts of the channel that cannot be modeled in the deterministic mode are simulated by some physical clusters through appropriate statistical distributions. This channel model has a very low 
computational time in comparison with full deterministic models, since it uses image theory instead of meshing the propagation environment to find the paths between Tx and $\mathrm{Rx}$. In addition, it employs statistic distributions to model complex parts of the channel and reduce computational time and memory. The accuracy of the model is high, because the main structures of the propagation channel are modeled in the deterministic mode and other complex parts of the channel are mapped with corresponding clusters. This approach has been applied to an indoor environment where the field measurements have been reported. Then, simulation results have been compared with measurement results to evaluate the accuracy of the proposed algorithm. A comparison between simulation and measurement shows that total behavior of the simulated channel follows measurements results.

\section{REFERENCES}

[1] S. Hur, S. Baek, B. Kim, et al. "Proposal on millimeter-wave channel Modeling for 5G cellular system," IEEE J. Sel. Topics in Signal Processing, vol. 10, no. 3, pp. 454-469, 2016.

[2] F. Boccadir, R. W. Heath, A. Lozano, et al. "Five disruptive technology directions for 5G," IEEE Commun., Mag., vol. 52, no. 2, pp. 74-80, 2014.

[3] A. Osseiran, F. Boccardi, V. Braun, et al. "Scenarios for 5G mobile and wireless communications: the vision of METIS project," IEEE Commun. Mag., vol. 52, no.5, pp. 26-35 May 2014.

[4] C.-X. Wang, F. Haider, X. Gao, et al. "Cellular architecture and key technologies for $5 \mathrm{G}$ wireless communication networks," IEEE Commun., Mag., vol. 52, no. 2, pp. 122-130 Feb. 2014.

[5] G. Andrews, S. Buzzi, W. Chol, et al. "What will 5G be?," IEEE J. Sel. Areas in Commun., vol. 32, no. 6, pp. 1065-1082, , June 2014.

[6] A. L. Swindlehurst, E. Ayanoglu, P. Heydari, et al. "Millimeter-wave massive MIMO: the next wireless revolution?," IEEE Commun. Mag., vol. 52, no. 9, pp. 56-62, Sep. 2014.

[7] E. G. Larsson, O. Edfors, F. Tufvesson, et al. "Massive MIMO for next generation wireless systems," IEEE Commun. Mag., vol. 52, no. 2, pp. 186-195, Feb. 2014.

[8] L. Lu, G. Y. Li, A. L. Swindlehurst, et al. "An overview of massive MIMO: benefits and challenges," IEEE J. Sel. topics in signal processing, vol. 8, no. 5, pp. 742-758, Oct. 2014.

[9] F. Rusek, D. Persson, B. K. Lau, et al. "Scaling up MIMO: opportunities and challenges with very large arrays," IEEE signal processing Mag., vol. 30, no. 1, pp. 40-60, Jan. 2013.

[10] K. Zheng, S. Ou, X., Yin, "Massive MIMO channel models: a survey," Int. J. of Antennas and Propag., vol. 2014. pp .1-10, June 2014.

[11] P. P. Tayade, V. M. Rohokale, "Enhancement of spectral efficiency, coverage and channel capacity for wireless communication towards 5G," Int. Conf. on Pervasive Computing (ICPC), pp. 1-5, Apr. 2015.

[12] W. -P. Zhu, S. Kusaladharma, W. Ajib, "Stochastic geometry-based modeling and analysis of massive MIMO-enabled millimeter wave cellular networks," IEEE Trans. on Commun., pp. 1-14, Sep. 2018,

[13] I. Khan, M. Singh, D. Singh, "Compressive sensing-based sparsity adaptive channel estimation for 5G massive MIMO systems," Applied Sciences (2076-3417), vol. 8, no. 5, pp. 1-13, May 2018.

[14] W. Fan, I. Carton, P. Kyosti, et al. "A step toward 5G in 2020: lowcost OTA performance evaluation of massive MIMO base stations," IEEE Antennas and Propag. Mag., vol. 59, no. 1, pp. 38-47, Feb. 2017.

[15] J. Hoidis, S. T. Brink, M. Debbah, "Massive MIMO in the UL/DL of cellular networks: how many antennas do we need? , IEEE J. Sel. Areas in Commun., vol. 31, no. 2, pp. 160-171, Feb. 2013.
[16] V. Jungnickel, K. Manolakin, W. Zirwas, et al. "The role of small cells, coordinated multipoint, and massive MIMO in 5G," IEEE Commun. Mag., vol. 52, no. 5, pp. 44-51, May 2014.

[17] C.-X. Wang, S. Wu, L. Bai, et al. "Recent advances and future challenges for massive MIMO channel measurements and models," Science China Inf. Sciences, vol. 59, no. 2, pp. 1-16, 2016.

[18] S. Wu, C.-X. Wang, M. M. Alwakeel, et al. "A non-stationary 3-D wideband twin-cluster model for 5G massive MIMO channels," IEEE J. Sel. Areas in Commun., vol. 32, no. 6, pp. 1207-1218, June 2014.

[19] X. Gao, O. Edfors, F., Rusek, et al. "Massive MIMO performance evaluation based on measured propagation data," IEEE Trans. Wireless Commun., vol. 14, no. 7, p. 3899-3911, July 2015.

[20] S. Payami, F. Tufvesson, "Channel measurements and analysis for very large array systems at $2.6 \mathrm{GHz}$," Europ. Conf. on Antennas and Propag.(EUCAP), pp. 433-437, Mar. 2012.

[21] T. S. Rappaport, S. Sun, R. Mayzus, et al. "Millimeter wave mobile communications for $5 \mathrm{G}$ cellular: it will work! ," IEEE access, vol. 1, no. 1, pp. 335-349, May 2013.

[22] T. Kim, J. Park, J.-Y. Seol, et al. Tens of Gbps support with mmWave beamforming systems for next generation communications," IEEE Global Commun. Conf.(GLOBECOM), pp. 3685-3690, 2013

[23] Y. Liu, L. Wang, S. A. R. Zaidi, et al. "Secure D2D communication in large-scale cognitive cellular networks: a wireless power transfer model," IEEE Trans. on Commun., vol. 64, no. 1, pp. 329-342, 2016.

[24] C.-X, Wang, J. Bian, J. Sun, et al. "A survey of 5G channel measurements and models," IEEE Commun. Surveys \& Tutorials, vol. 20, no. 4, pp.3142-3168, Aug. 2018.

[25] S. Wu, C.-X. Wang, M. M. Alwakeel, "A general 3D non-stationary 5G wireless channel model," IEEE Trans. on Commun., vol. 66 , no.7, pp. 3065-3078, July 2018.

[26] S. A. Busari, K. M. S. Huq, S. Mumtaz, et al. "Millimeter-wave massive MIMO communication for future wireless systems: a survey," IEEE Commun. Surveys \& Tutorials, vol. 20, no. 2, pp. 836-869, Sep. 2017.

[27] S. L. H. Nguyen, K. Haneda, J. Jarvelainen, et al. "On the mutual orthogonality of millimeter-wave massive MIMO channels," IEEE Veh. Tech. Conf. (VTC Spring), pp. 1-5, 2015.

[28] S. Mumtaz, J. Rodriguez, L. Dai, "Introduction to mmWave massive MIMO a paradigm for 5G," Academic Press, (Chapter one), pp. 118: Elsevier, 2017.

[29] IST-WINNER, Deliverable 1.1. 2 v. 1.2. "WINNER II channel models," IST-WINNER II, Tech. Rep., 2007.

[30] J. Meinila, P. Kyosti, L. Hentila, et al. D5. 3: "WINNER+ final channel models," Wireless World Initiative New Radio, 2010.

[31] C. Lopez, C.-X. Wang, "Novel 3-D non-Stationary wideband models for massive MIMO channels," IEEE Trans. on Wireless Commun. vol. 17, no. 5, pp. 2893-2905, May 2018

[32] Y. Liu, C.-X. Wang, J. Huang, et al. "Novel 3D non-stationary mmWave massive MIMO channel models for 5G high-speed train wireless communications," In proc. IEEE Trans on Veh. Tech., pp. 1-11, Aug. 2018.

[33] J. Li, B., Ai, R., He, et al. "Cluster-based 3-D channel modeling for massive MIMO in subway station environment," IEEE Access, vol. 6, pp. 6257-6272, 2018.

[34] Y. Li, R., He, S., Lin, et al. "Cluster-based nonstationary channel modeling for vehicle-to-vehicle communications," IEEE Antennas and Wireless Propag. Letters, vol. 16, pp. 1419-1422, Nov. 2017.

[35] P. Zhang, H. Wang, X., Sun, et al. "Cluster-based analysis of wideband millimeter-wave channel for corridor environment," Sixth Asia-Pacific Conf. on Antennas and Propag. (APCAP), pp. 1-3, Oct. 2017.

[36] J. Yao, H. Ren, Q. Liu, "Massive MIMO channel modeling using map-based ray tracing method," IEEE Int. Symp. on Microwave, Antenna, Propag., and EMC Tech. (MAPE), pp. 1-5, 2017.

[37] L. Bai, C.-X. Wang, S. Wu, et al. "A 3-D wideband multi-confocal ellipsoid model for wireless massive MIMO communication channels with uniform planar antenna array," IEEE 85th Veh. Tech. Conf. (VTC Spring), pp. 1-6, June 2017.

[38] M. M. Tamaddondar, N. Noori, "Cluster based massive MIMO channel model for indoor environment using ray tracing technique," 
IEEE Asia Pacific Microwave Conf. (APMC), pp. 1030-1033, Nov. 2017.

[39] J. Chen, X. Yin, X. Cai, et al. "Measurement-based massive MIMO channel modeling for outdoor LoS and NLoS environments," IEEE Access, vol. 5, no. 99, pp. 2126-2140, Jan. 2017.

[40] J. Huang, C.-X. Wang, R. Feng, et al. "Multi-frequency mmWave massive MIMO channel measurements and characterization for 5G wireless communication systems," IEEE J. Sel. Areas in Commun., vol. 35, no. 7, pp. 1591-1605, July 2017.

[41] Y. Chen, Y. Li, S., Sun, et al. "A twin-multi-ring channel model for Massive MIMO system," Int. Symp. on Commun. and Inf. Tech. (ISCIT), pp. 606-610, 2016.

[42] S. Wu, C.-X. Wang, H. Haas, et al. "A non-stationary wideband channel model for massive MIMO communication systems," IEEE Trans. on wireless Commun., vol. 14, no. 3, pp. 1434-1446, Mar. 2015

[43] V. Nurmela, A. Karttunen, A. Roivainen, et al. "METIS channe models," FP7 METIS, Deliverable D1.4, vol. 1, , July 2015.
[44] H. Jiang, Z. Zhang, J. Dang, et al. "A novel 3-D massive MIMO channel model for vehicle-to-vehicle communication environments," IEEE Trans. on Commun., vol. 66, no. 1, pp. 79-90, , Jan. 2018.

[45] X. Cheng, Y., He, Channel modeling and analysis of ULA massive MIMO systems," Int. Conf. on Advanced Commun. Tech. (ICACT), pp. 411-416, 2018.

[46] B. Ai, K. Guan, R. He, et al. "On indoor millimeter wave massive MIMO channels: measurement and simulation, "IEEE J. on Sel. Areas in Commun., Vol. 35, No. 7, pp. 1678-1690, July 2017.

[47] E. Bonek, W. Weichselberger, M. Herdin, et al. "A geometry-based stochastic MIMO channel model for $4 \mathrm{G}$ indoor broadband packet access, " Citeseer, 2005.

[48] M. R. Akdenizk, Y. Liu, M. K. Samimi, et al. "Millimeter Wave Channel Modeling and Cellular Capacity Evaluation, "IEEE J. on Sel. Areas in Commun., vol. 32, no. 6, pp. 1164-1179, June 2014

[49] A. Abdi, M. Kaveh, "A space-time correlation model for multielement antenna systems in mobile fading channels, "IEEE $J$. on Sel. Areas in Commun., vol. 20, no. 3, pp. 550-560, 2002. 